\title{
Existence Results for Fractional Differential Inclusions with Multivalued Term Depending on Lower-Order Derivative
}

\author{
Xiaoyou Liu' ${ }^{1}$ and Zhenhai Liu ${ }^{2}$ \\ ${ }^{1}$ School of Mathematical Science and Computing Technology, Central South University, \\ Changsha, Hunan 410075, China \\ 2 School of Science, Guangxi University for Nationalities, Nanning, Guangxi 530006, China \\ Correspondence should be addressed to Xiaoyou Liu, liuxiaoyou2002@hotmail.com
}

Received 24 October 2012; Accepted 14 December 2012

Academic Editor: Svatoslav Staněk

Copyright (C) 2012 X. Liu and Z. Liu. This is an open access article distributed under the Creative Commons Attribution License, which permits unrestricted use, distribution, and reproduction in any medium, provided the original work is properly cited.

This paper is concerned with a class of fractional differential inclusions whose multivalued term depends on lower-order fractional derivative with fractional (non)separated boundary conditions. The cases of convex-valued and non-convex-valued right-hand sides are considered. Some existence results are obtained by using standard fixed point theorems. A possible generalization for the inclusion problem with integral boundary conditions is also discussed. Examples are given to illustrate the results.

\section{Introduction}

Recently, the subject of fractional differential equations has emerged as an important area of investigation. Indeed, we can find numerous applications of fractional-order derivatives in the mathematical modeling of physical and biological phenomena in various fields of science and engineering [1-3]. A variety of results on initial and boundary value problems of fractional differential equations and inclusions can easily be found in the literature on this topic. For some recent results, we can refer to, for instance, [4-20] (equations) [21-27] (inclusions) and the references therein.

Ahmad and Ntouyas [22] considered a boundary value problem of fractional differential inclusions with fractional separated boundary conditions given by

$$
\begin{aligned}
{ }^{c} D^{q} x(t) & \in F(t, x(t)), \quad t \in[0,1], 1<q \leq 2, \\
\alpha_{1} x(0)+\beta_{1}\left({ }^{c} D^{p} x(0)\right) & =\gamma_{1}, \quad \alpha_{2} x(1)+\beta_{2}\left({ }^{c} D^{p} x(1)\right)=\gamma_{2}, \quad 0<p<1,
\end{aligned}
$$


where ${ }^{c} D^{q}$ denotes the Caputo fractional derivative of order $q, F:[0,1] \times \mathbb{R} \rightarrow 2^{\mathbb{R}}$ is a multivalued map, and $\alpha_{i}, \beta_{i}, \gamma_{i}(i=1,2)$ are real constants, with $\alpha_{1} \neq 0$.

In Cernea [24], the following multipoint boundary value problem for a fractionalorder differential inclusion was studied

$$
\begin{gathered}
D^{\alpha} x(t) \in F\left(t, x(t), x^{\prime}(t)\right) \quad \text { a.e. } t \in[0,1], 2<\alpha \leq 3, \\
x(0)=x^{\prime}(0)=0, \quad x(1)-\sum_{i=1}^{m} a_{i} x\left(\xi_{i}\right)=\lambda,
\end{gathered}
$$

where $D^{\alpha}$ is the standard Riemann-Liouville fractional derivative, $m \geq 1,0<\xi_{1}<\xi_{2}<\cdots<$ $\xi_{m}<1, \sum_{i=1}^{m} a_{i} \xi_{i}^{\alpha-1}<1, \lambda>0, a_{i}>0, i=1,2, \ldots, m$, and $F:[0,1] \times \mathbb{R} \times \mathbb{R} \rightarrow 2^{\mathbb{R}}$ is a multivalued map.

In Khan et al. [11], the authors studied the existence and uniqueness results of nonlinear fractional differential equation of the type

$$
\begin{gathered}
{ }^{c} D^{q} x(t)=f\left(t, x(t),{ }^{c} D^{\sigma} x(t)\right), \quad t \in[0, T], \\
\alpha x(0)-\beta x^{\prime}(0)=\int_{0}^{T} g(s, x) d s, \quad \gamma x(T)+\delta x^{\prime}(T)=\int_{0}^{T} h(s, x) d s,
\end{gathered}
$$

where $0<\sigma<1,1<q<2, \alpha, \delta>0, \beta, \gamma \geq 0$ (or $\alpha, \delta \geq 0, \beta, \gamma>0$ ) and ${ }^{c} D^{q},{ }^{c} D^{\sigma}$ are the Caputo fractional derivatives. The results in $[11,22,24]$ are obtained by using appropriate standard fixed point theorems.

Motivated by the papers cited above, in this paper, we consider the existence results for a new class of fractional differential inclusions of the form

$$
{ }^{c} D^{\alpha} x(t) \in F\left(t, x(t),{ }^{c} D^{\beta} x(t)\right), \quad \text { a.e. } t \in[0, T],
$$

where ${ }^{c} D^{\alpha}$ denotes the Caputo fractional derivative of order $\alpha, F:[0,1] \times \mathbb{R} \times \mathbb{R} \rightarrow 2^{\mathbb{R}}$ is a multivalued map, $1<\alpha \leq 2,0<\beta \leq 1$, and $T>0$. We study (1.4) subject to two families of boundary conditions:

(1) separated boundary conditions

$$
a_{1} x(0)+b_{1}\left({ }^{c} D^{\gamma} x(0)\right)=c_{1}, \quad a_{2} x(T)+b_{2}\left({ }^{c} D^{\gamma} x(T)\right)=c_{2},
$$

(2) Nonseparated boundary conditions

$$
a_{1} x(0)+b_{1} x(T)=c_{1}, \quad a_{2}\left({ }^{c} D^{\gamma} x(0)\right)+b_{2}\left({ }^{c} D^{\gamma} x(T)\right)=c_{2},
$$

where $a_{i}, b_{i}, c_{i}, i=1,2$ are real constants and $0<\gamma<1$. 
The results of this paper can easily to be generalized to the boundary value problems of fractional differential inclusions (1.4) with the following integral boundary conditions:

$$
\begin{gathered}
a_{1} x(0)+b_{1}\left({ }^{c} D^{\gamma} x(0)\right)=c_{1} \int_{0}^{T} g(s, x(s)) d s, \\
a_{2} x(T)+b_{2}\left({ }^{c} D^{\gamma} x(T)\right)=c_{2} \int_{0}^{T} h(s, x(s)) d s, \\
a_{1} x(0)+b_{1} x(T)=c_{1} \int_{0}^{T} g(s, x(s)) d s, \\
a_{2}\left({ }^{c} D^{\gamma} x(0)\right)+b_{2}\left({ }^{c} D^{\gamma} x(T)\right)=c_{2} \int_{0}^{T} h(s, x(s)) d s,
\end{gathered}
$$

where $g, h:[0, T] \times \mathbb{R} \rightarrow \mathbb{R}$ are given functions.

We remark that when the third variable of the multifunction $F$ in (1.4) vanishes, the problem (1.4), (1.5) reduces to the case considered in [22]. When $a_{1}=b_{1}=1, a_{2}=b_{2}=1$, and $c_{1}=c_{2}=0$, the problem (1.4), (1.6) reduces to an antiperiodic fractional boundary value problem (the case of $F=f$ a given continuous function was studied in [4, 15]). Our results generalize some results from the literature cited above and constitute a contribution to this emerging field of research.

The rest of the paper is organized as follows: in Section 2 we present the notations and definitions and give some preliminary results that we need in the sequel, Section 3 is dedicated to the existence results of the fractional differential inclusion (1.4) with boundary conditions (1.5) and (1.6), in Section 4 we indicate a possible generalization for the inclusion problem (1.4) with integral boundary conditions (1.7) and (1.8), and two illustrative examples are given in Section 5.

\section{Preliminaries}

In this section, we introduce notations, definitions, and preliminary facts that will be used in the remainder of this paper.

Let $(X,\|\cdot\|)$ be a normed space. We use the notations $P(X)=\{Y \subseteq X: Y \neq \emptyset\}, P_{\mathrm{cl}}(X)=$ $\{Y \in P(X): Y$ closed $\}, P_{b}(X)=\{Y \in P(X): Y$ bounded $\}, P_{\text {cp }}(X)=\{Y \in P(X): Y$ compact $\}$, $P_{\mathrm{cp}, \mathrm{c}}(X)=\{Y \in P(X): Y$ compact, convex $\}$, and so on.

Let $A, B \in P_{\mathrm{cl}}(\mathrm{X})$; the Pompeiu-Hausdorff distance of $A, B$ is defined as

$$
h(A, B)=\max \left\{\sup _{a \in A} d(a, B), \sup _{b \in B} d(b, A)\right\} .
$$

A multivalued map $F: X \rightarrow P(X)$ is convex (closed) valued if $F(x)$ is convex (closed) for all $x \in X$. $F$ is said to be completely continuous if $F(B)$ is relatively compact for every $B \in P_{b}(X) . F$ is called upper semicontinuous on $X$, if for every $x_{0} \in X$, the set $F\left(x_{0}\right)$ is a nonempty closed subset of $X$, and for every open set $O$ of $X$ containing $F\left(x_{0}\right)$, there exists an open neighborhood $U_{0}$ of $x_{0}$ such that $F\left(U_{0}\right) \subseteq O$. Equivalently, $F$ is upper semicontinuous if the set $\{x \in X: F(x) \subseteq O\}$ is open for any open set $O$ of $X$. $F$ is called lower semicontinuous 
if the set $\{x \in X: F(x) \cap O \neq \emptyset\}$ is open for each open set $O$ in $X$. If a multivalued map $F$ is completely continuous with nonempty compact values, then $F$ is upper semicontinuous if and only if $F$ has a closed graph, that is, if $x_{n} \rightarrow x_{*}$ and $y_{n} \rightarrow y_{*}$, then $y_{n} \in F\left(x_{n}\right)$ implies $y_{*} \in F\left(x_{*}\right)[28]$.

A multivalued map $F:[0, T] \rightarrow P_{\mathrm{cl}}(X)$ is said to be measurable if, for every $x \in X$, the function $t \rightarrow d(x, F(t))=\inf \{d(x, y): y \in F(t)\}$ is a measurable function.

Definition 2.1. A multivalued map $F: X \rightarrow P_{\mathrm{cl}}(X)$ is called

(1) $\gamma$-Lipschitz if there exists $\gamma>0$ such that

$$
h(F(x), F(y)) \leq \gamma d(x, y), \quad \text { for each } x, y \in X,
$$

(2) a contraction if it is $\gamma$-Lipschitz with $\gamma<1$.

Definition 2.2. A multivalued map $F:[0, T] \times \mathbb{R} \times \mathbb{R} \rightarrow P(\mathbb{R})$ is said to be Carathéodory if

(1) $t \rightarrow F(t, x, y)$ is measurable for each $x, y \in \mathbb{R}$;

(2) $(x, y) \rightarrow F(t, x, y)$ is upper semicontinuous for a.e. $t \in[0, T]$.

Further, a Carathéodory function $F$ is said to be $L^{1}$ - Carathéodory if

(3) for each $l>0$, there exists $\varphi_{l} \in L^{1}\left([0, T], \mathbb{R}^{+}\right)$such that

$$
\|F(t, x, y)\|=\sup \{|v|: v \in F(t, x, y)\} \leq \varphi_{l}(t)
$$

for all $|x| \leq l,|y| \leq l$ and a.e. $t \in[0, T]$.

Lemma 2.3 (see [29]). Let $X$ be a Banach space. Let $G:[0, T] \times X \rightarrow P_{c p, c}(X)$ be an $L^{1}$ Carathéodory multivalued map and $\Gamma$ a linear continuous map from $L^{1}([0, T], X)$ to $C([0, T], X)$, then the operator

$$
\Gamma \circ S_{G}: C([0, T], X) \longrightarrow P_{c p, c}(C([0, T], X)), \quad y \longmapsto\left(\Gamma \circ S_{G}\right)(y)=\Gamma\left(S_{G, y}\right)
$$

is a closed graph operator in $C([0, T], X) \times C([0, T], X)$.

Here $S_{G, y}=\left\{v \in L^{1}([0, T], X): v(t) \in G(t, y(t))\right.$ for a.e. $\left.t \in[0, T]\right\}$.

Definition 2.4 (see [30]). The Riemann-Liouville fractional integral of order $q$ for a function $f$ is defined as

$$
I^{q} f(t)=\frac{1}{\Gamma(q)} \int_{0}^{t} \frac{f(s)}{(t-s)^{1-q}} d s, \quad q>0
$$

provided the integral exists. 
Definition 2.5 (see [30]). For at least $n$-times differentiable function $f$, the Caputo derivative of order $q$ is defined as

$$
{ }^{c} D^{q} f(t)=\frac{1}{\Gamma(n-q)} \int_{0}^{t}(t-s)^{n-q-1} f^{(n)}(s) d s, \quad n-1<q<n, n=[q]+1,
$$

where $[q]$ denotes the integer part of the real number $q$.

Lemma 2.6 (see [20]). Let $\alpha>0$; then the differential equation

$$
{ }^{c} D^{\alpha} h(t)=0
$$

has solutions $h(t)=c_{0}+c_{1} t+c_{2} t^{2}+\cdots+c_{n-1} t^{n-1}$ and

$$
I^{\alpha c} D^{\alpha} h(t)=h(t)+c_{0}+c_{1} t+c_{2} t^{2}+\cdots+c_{n-1} t^{n-1}
$$

here $c_{i} \in \mathbb{R}, i=0,1,2, \ldots, n-1, n=[\alpha]+1$.

The following lemma obtained in [6] is useful in the rest of the paper.

Lemma 2.7 (see [6]). For a given $y \in C([0, T], \mathbb{R})$, the unique solution of the fractional separated boundary value problem

$$
\begin{gathered}
{ }^{c} D^{\alpha} x(t)=y(t), \quad t \in[0, T], 1<\alpha \leq 2, \\
a_{1} x(0)+b_{1}\left({ }^{c} D^{\gamma} x(0)\right)=c_{1}, \quad a_{2} x(T)+b_{2}\left({ }^{c} D^{\gamma} x(T)\right)=c_{2}, \quad 0<\gamma<1,
\end{gathered}
$$

is given by

$$
\begin{aligned}
x(t)= & \int_{0}^{t} \frac{(t-s)^{\alpha-1}}{\Gamma(\alpha)} y(s) d s \\
& -\frac{t}{v_{1}}\left(a_{2} \int_{0}^{T} \frac{(T-s)^{\alpha-1}}{\Gamma(\alpha)} y(s) d s+b_{2} \int_{0}^{T} \frac{(T-s)^{\alpha-\gamma-1}}{\Gamma(\alpha-\gamma)} y(s) d s\right)+v_{2} t+\frac{c_{1}}{a_{1}},
\end{aligned}
$$

where

$$
v_{1}=\frac{a_{2} T \Gamma(2-\gamma)+b_{2} T^{1-\gamma}}{\Gamma(2-\gamma)}, \quad v_{2}=\frac{a_{1} c_{2}-a_{2} c_{1}}{a_{1} v_{1}}
$$

We notice that the solution (2.10) of the problem (2.9) does not depend on the parameter $b_{1}$, that is to say, the parameter $b_{1}$ is of arbitrary nature for this problem. And by (2.10), we should assume that $a_{1} \neq 0$ and $a_{2} \operatorname{Tr}^{\gamma}(2-\gamma) \neq-b_{2}$. 
Lemma 2.8. For any $y \in C([0, T], \mathbb{R})$, the unique solution of the fractional nonseparated boundary value problem

$$
\begin{gathered}
{ }^{c} D^{\alpha} x(t)=y(t), \quad t \in[0, T], 1<\alpha \leq 2, \\
a_{1} x(0)+b_{1} x(T)=c_{1}, \quad a_{2}\left({ }^{c} D^{\gamma} x(0)\right)+b_{2}\left({ }^{c} D^{\gamma} x(T)\right)=c_{2}, \quad 0<\gamma<1,
\end{gathered}
$$

is given by

$$
\begin{aligned}
x(t)= & \int_{0}^{t} \frac{(t-s)^{\alpha-1}}{\Gamma(\alpha)} y(s) d s-\frac{t \Gamma(2-\gamma)}{T^{1-\gamma}} \int_{0}^{T} \frac{(T-s)^{\alpha-\gamma-1}}{\Gamma(\alpha-\gamma)} y(s) d s+\frac{t \Gamma(2-\gamma) c_{2}}{T^{1-\gamma} b_{2}} \\
& -\frac{b_{1}}{a_{1}+b_{1}}\left(\int_{0}^{T} \frac{(T-s)^{\alpha-1}}{\Gamma(\alpha)} y(s) d s-T^{\gamma} \Gamma(2-\gamma) \int_{0}^{T} \frac{(T-s)^{\alpha-\gamma-1}}{\Gamma(\alpha-\gamma)} y(s) d s\right) \\
& -\frac{1}{a_{1}+b_{1}}\left(\frac{b_{1} c_{2} T^{\gamma} \Gamma(2-\gamma)}{b_{2}}-c_{1}\right) .
\end{aligned}
$$

Proof. For $1<\alpha \leq 2$, by Lemma 2.6, we know that the general solution of the equation ${ }^{c} D^{\alpha} x(t)=y(t)$ can be written as

$$
x(t)=I^{\alpha} y(t)-k_{1}-k_{2} t=\int_{0}^{t} \frac{(t-s)^{\alpha-1}}{\Gamma(\alpha)} y(s) d s-k_{1}-k_{2} t
$$

where $k_{1}, k_{2} \in \mathbb{R}$ are arbitrary constants. Since ${ }^{c} D^{\gamma} k=0$ ( $k$ is a constant $),{ }^{c} D^{\gamma} t=t^{1-\gamma} / \Gamma(2-\gamma)$, ${ }^{c} D^{\gamma} I^{\alpha} y(t)=I^{\alpha-\gamma} y(t)$ (see [30]), from (2.14), we have

$$
{ }^{c} D^{\gamma} x(t)=I^{\alpha-\gamma} y(t)-\frac{k_{2} t^{1-\gamma}}{\Gamma(2-\gamma)}=\int_{0}^{t} \frac{(t-s)^{\alpha-\gamma-1}}{\Gamma(\alpha-\gamma)} y(s) d s-\frac{k_{2} t^{1-\gamma}}{\Gamma(2-\gamma)} .
$$

Using the boundary conditions, we obtain

$$
\begin{aligned}
& a_{1}\left(-k_{1}\right)+b_{1}\left(\int_{0}^{T} \frac{(T-s)^{\alpha-1}}{\Gamma(\alpha)} y(s) d s-k_{1}-k_{2} T\right)=c_{1}, \\
& a_{2} \times 0+b_{2}\left(\int_{0}^{T} \frac{(T-s)^{\alpha-\gamma-1}}{\Gamma(\alpha-\gamma)} y(s) d s-\frac{k_{2} T^{1-\gamma}}{\Gamma(2-\gamma)}\right)=c_{2} .
\end{aligned}
$$


Therefore, we have

$$
\begin{gathered}
k_{1}=\frac{1}{a_{1}+b_{1}}\left(\frac{b_{1} c_{2} T^{\gamma} \Gamma(2-\gamma)}{b_{2}}-c_{1}\right) \\
+\frac{b_{1}}{a_{1}+b_{1}}\left(\int_{0}^{T} \frac{(T-s)^{\alpha-1}}{\Gamma(\alpha)} y(s) d s-T^{\gamma} \Gamma(2-\gamma) \int_{0}^{T} \frac{(T-s)^{\alpha-\gamma-1}}{\Gamma(\alpha-\gamma)} y(s) d s\right), \\
k_{2}=\frac{\Gamma(2-\gamma)}{T^{1-\gamma}}\left(\int_{0}^{T} \frac{(T-s)^{\alpha-\gamma-1}}{\Gamma(\alpha-\gamma)} y(s) d s-\frac{c_{2}}{b_{2}}\right) .
\end{gathered}
$$

Substituting the values of $k_{1}, k_{2}$ in (2.14), we obtain (2.13). This completes the proof.

From the proof of the above lemma, we notice that the solution (2.13) of the problem (2.12) does not depend on the parameter $a_{2}$, that is to say, the parameter $a_{2}$ is of arbitrary nature for this problem. In this situation, we need to assume that $a_{1}+b_{1} \neq 0$ and $b_{2} \neq 0$.

Let us define what we mean by a solution of the problem (1.4), (1.5) and the problem (1.4), (1.6).

Definition 2.9. A function $x \in A C^{1}([0, T], \mathbb{R})$ is a solution of the problem (1.4), (1.5) if it satisfies the boundary conditions (1.5) and there exists a function $f \in L^{1}([0, T], \mathbb{R})$ such that $f(t) \in F\left(t, x(t),{ }^{c} D^{\beta} x(t)\right)$ a.e. on $t \in[0, T]$ and

$$
\begin{aligned}
x(t)= & \int_{0}^{t} \frac{(t-s)^{\alpha-1}}{\Gamma(\alpha)} f(s) d s \\
& -\frac{t}{v_{1}}\left(a_{2} \int_{0}^{T} \frac{(T-s)^{\alpha-1}}{\Gamma(\alpha)} f(s) d s+b_{2} \int_{0}^{T} \frac{(T-s)^{\alpha-\gamma-1}}{\Gamma(\alpha-\gamma)} f(s) d s\right)+v_{2} t+\frac{c_{1}}{a_{1}} .
\end{aligned}
$$

Definition 2.10. A function $x \in A C^{1}([0, T], \mathbb{R})$ is a solution of the problem (1.4), (1.6) if it satisfies the boundary conditions (1.6) and there exists a function $f \in L^{1}([0, T], \mathbb{R})$ such that $f(t) \in F\left(t, x(t),{ }^{c} D^{\beta} x(t)\right)$ a.e. on $t \in[0, T]$ and

$$
\begin{aligned}
x(t)= & \int_{0}^{t} \frac{(t-s)^{\alpha-1}}{\Gamma(\alpha)} f(s) d s-\frac{t \Gamma(2-\gamma)}{T^{1-\gamma}} \int_{0}^{T} \frac{(T-s)^{\alpha-\gamma-1}}{\Gamma(\alpha-\gamma)} f(s) d s+\frac{t \Gamma(2-\gamma) c_{2}}{T^{1-\gamma} b_{2}} \\
& -\frac{b_{1}}{a_{1}+b_{1}}\left(\int_{0}^{T} \frac{(T-s)^{\alpha-1}}{\Gamma(\alpha)} f(s) d s-T^{\gamma} \Gamma(2-\gamma) \int_{0}^{T} \frac{(T-s)^{\alpha-\gamma-1}}{\Gamma(\alpha-\gamma)} f(s) d s\right) \\
& -\frac{1}{a_{1}+b_{1}}\left(\frac{b_{1} c_{2} T^{\gamma} \Gamma(2-\gamma)}{b_{2}}-c_{1}\right) .
\end{aligned}
$$

Let $C([0, T], \mathbb{R})$ be the space of all continuous functions defined on $[0, T]$. Define the space $x=\left\{x: x\right.$ and $\left.{ }^{c} D^{\beta} x \in C([0, T], \mathbb{R})\right\}(0<\beta \leq 1)$ endowed with the norm $\|x\|=$ $\max _{t \in[0, T]}|x(t)|+\left.\max _{t \in[0, T]}\right|^{c} D^{\beta} x(t) \mid$. We know that $(\mathcal{X},\|\cdot\|)$ is a Banach space (see [14]).

We end this section with two fixed point theorems, which will be used in the sequel. 
Theorem 2.11 (nonlinear alternative of Leray-Schauder type [31]). Let X be a Banach space, C a closed convex subset of $X, U$ an open subset of $C$ with $0 \in U$. Suppose that $F: \bar{U} \rightarrow P_{c p, c}(C)$ is an upper semicontinuous compact map. Then either (1) F has a fixed point in $\bar{U}$, or (2) there is a $x \in \partial U$ and $\lambda \in(0,1)$ such that $x \in \lambda F(x)$.

Theorem 2.12 (Covitz and Nadler Jr. [32]). Let $(X, d)$ be a complete metric space. If $F: X \rightarrow$ $P_{c l}(X)$ is a contraction, then $F$ has a fixed point.

\section{Existence Results}

In this section, we will give some existence results for the problems (1.4), (1.5) and (1.4), (1.6).

For each $x \in \mathcal{X}$, define the set of selections of $F$ by

$$
S_{F, x}=\left\{v \in L^{1}([0, T], \mathbb{R}): v(t) \in F\left(t, x(t),{ }^{c} D^{\beta} x(t)\right) \text { for a.e. } t \in[0, T]\right\}
$$

In view of Lemmas 2.7 and 2.8 , we define operators $N, M: \mathcal{X} \rightarrow P(\mathcal{X})$ as

$$
\begin{aligned}
& N(x)=\left\{h \in \mathcal{X}: h(t)=(S u)(t), u \in S_{F, x}\right\} \\
& M(x)=\left\{h \in \mathcal{X}: h(t)=(K u)(t), u \in S_{F, x}\right\}
\end{aligned}
$$

with

$$
\begin{aligned}
(S u)(t)= & \int_{0}^{t} \frac{(t-s)^{\alpha-1}}{\Gamma(\alpha)} u(s) d s \\
& -\frac{t}{v_{1}}\left(a_{2} \int_{0}^{T} \frac{(T-s)^{\alpha-1}}{\Gamma(\alpha)} u(s) d s+b_{2} \int_{0}^{T} \frac{(T-s)^{\alpha-\gamma-1}}{\Gamma(\alpha-\gamma)} u(s) d s\right)+v_{2} t+\frac{c_{1}}{a_{1}}, \\
(K u)(t)= & \int_{0}^{t} \frac{(t-s)^{\alpha-1}}{\Gamma(\alpha)} u(s) d s-\frac{t \Gamma(2-\gamma)}{T^{1-\gamma}} \int_{0}^{T} \frac{(T-s)^{\alpha-\gamma-1}}{\Gamma(\alpha-\gamma)} u(s) d s+\frac{t \Gamma(2-\gamma) c_{2}}{T^{1-\gamma} b_{2}} \\
& -\frac{b_{1}}{a_{1}+b_{1}}\left(\int_{0}^{T} \frac{(T-s)^{\alpha-1}}{\Gamma(\alpha)} u(s) d s-T^{\gamma} \Gamma(2-\gamma) \int_{0}^{T} \frac{(T-s)^{\alpha-\gamma-1}}{\Gamma(\alpha-\gamma)} u(s) d s\right) \\
& -\frac{1}{a_{1}+b_{1}}\left(\frac{b_{1} c_{2} T^{\gamma} \Gamma(2-\gamma)}{b_{2}}-c_{1}\right) .
\end{aligned}
$$

It is clear that if $x \in \mathcal{X}$ is a fixed point of the operator $N$ (the operator $M$ ), then $x$ is a solution of the problem (1.4), (1.5) (the problem (1.4), (1.6)).

Now we are in a position to present our main results. The methods used to prove the existence results are standard; however, their exposition in the framework of problems (1.4), (1.5) and (1.4), (1.6) is new. 


\subsection{Convex Case}

We consider first the case when $F$ is convex valued.

(H1): (1) $F:[0, T] \times \mathbb{R} \times \mathbb{R} \rightarrow P_{\mathrm{cp}, c}(\mathbb{R})$ is a Carathéodory multivalued map; (2) there exist $m \in L^{\infty}\left([0, T], \mathbb{R}^{+}\right)$and $\varphi, \psi:[0, \infty) \rightarrow(0, \infty)$ continuous, nondecreasing such that

$$
\|F(t, x, y)\|=\sup \{|v|: v \in F(t, x, y)\} \leq m(t)(\varphi(|x|)+\psi(|y|))
$$

for $x, y \in \mathbb{R}$ and a.e. $t \in[0, T]$.

Theorem 3.1. Assume that (H1) is satisfied and there exists L $>0$ such that

$$
\frac{L}{P+(\varphi(L)+\psi(L))\|m\|_{L^{\infty}} Q}>1
$$

where

$$
\begin{gathered}
P=\left|v_{2}\right| T+\frac{\left|c_{1}\right|}{\left|a_{1}\right|}+\frac{\left|v_{2}\right| T^{1-\beta}}{\Gamma(2-\beta)} \\
Q=\frac{T^{\alpha-\beta}}{\Gamma(\alpha-\beta+1)}+\frac{T^{\alpha}}{\Gamma(\alpha+1)}+\frac{T}{\left|v_{1}\right|}\left(1+\frac{T^{-\beta}}{\Gamma(2-\beta)}\right)\left(\frac{\left|a_{2}\right| T^{\alpha}}{\Gamma(\alpha+1)}+\frac{\left|b_{2}\right| T^{\alpha-\gamma}}{\Gamma(\alpha-\gamma+1)}\right) .
\end{gathered}
$$

Then the problem (1.4), (1.5) has at least one solution on $[0, T]$.

Proof. Consider the operator $N: \mathcal{X} \rightarrow P(\mathcal{X})$ defined by (3.2). From (H1), we have for each $x \in \mathcal{X}$, the set $S_{F, x}$ is nonempty [29]. For $x \in \mathcal{X}$, let $u \in S_{F, x}$ and $h=S u$, that is, $h \in N(x)$, we have

$$
{ }^{c} D^{\beta} h(t)=\int_{0}^{t} \frac{(t-s)^{\alpha-\beta-1}}{\Gamma(\alpha-\beta)} u(s) d s-\frac{k t^{1-\beta}}{\Gamma(2-\beta)},
$$

where $k$ is a constant given by

$$
k=\frac{1}{v_{1}}\left(a_{2} \int_{0}^{T} \frac{(T-s)^{\alpha-1}}{\Gamma(\alpha)} u(s) d s+b_{2} \int_{0}^{T} \frac{(T-s)^{\alpha-\gamma-1}}{\Gamma(\alpha-\gamma)} u(s) d s\right)-v_{2} .
$$

Hence we know that the operator $N: \mathcal{X} \rightarrow P(\mathcal{X})$ is well defined.

We put $S u=S_{1} u+S_{2} u$ where

$$
\left(S_{1} u\right)(t)=\int_{0}^{t} \frac{(t-s)^{\alpha-1}}{\Gamma(\alpha)} u(s) d s, \quad\left(S_{2} u\right)(t)=-k_{u} t+\frac{c_{1}}{a_{1}} .
$$

Here $k_{u}$ means that the constant $k$ defined by (3.9) is related to $u$. 
We will show that $N$ satisfies the requirements of the nonlinear alternative of LeraySchauder type. The proof will be given in five steps.

Step $1\left(N(x)\right.$ is convex valued). Since $F$ is convex valued, we know that $S_{F, x}$ is convex and therefore it is obvious that $N(x)$ is convex for each $x \in \mathcal{X}$.

Step 2 ( $N$ maps bounded sets into bounded sets in $\mathcal{X}$ ). Let $B_{r}$ be a bounded subset of $\mathcal{X}$ such that for any $x \in B_{r},\|x\| \leq r, r>0$. We prove that there exists a constant $l>0$ such that for each $x \in B_{r}$, one has $\|h\| \leq l$ for each $h \in N(x)$. Let $x \in B_{r}$ and $h \in N(x)$, then there exists $u \in S_{F, x}$ such that

$$
h(t)=(S u)(t) \quad \text { for } t \in[0, T] .
$$

By simple calculations, we have

$$
\begin{gathered}
\left|\left(S_{1} u\right)(t)\right| \leq \int_{0}^{t} \frac{(t-s)^{\alpha-1}}{\Gamma(\alpha)}|u(s)| d s \leq(\varphi(r)+\psi(r))\|m\|_{L^{\infty}} \frac{T^{\alpha}}{\Gamma(\alpha+1)}, \\
\left|\left(S_{2} u\right)(t)\right| \leq \frac{(\varphi(r)+\psi(r))\|m\|_{L^{\infty}} T}{\left|v_{1}\right|}\left(\frac{\left|a_{2}\right| T^{\alpha}}{\Gamma(\alpha+1)}+\frac{\left|b_{2}\right| T^{\alpha-\gamma}}{\Gamma(\alpha-\gamma+1)}\right)+\left|v_{2}\right| T+\frac{\left|c_{1}\right|}{\left|a_{1}\right|}, \\
\left|{ }^{c} D^{\beta} h(t)\right| \leq \int_{0}^{t} \frac{(t-s)^{\alpha-\beta-1}}{\Gamma(\alpha-\beta)}|u(s)| d s+\frac{\left|k_{u}\right| T^{1-\beta}}{\Gamma(2-\beta)} \\
\leq(\varphi(r)+\psi(r))\|m\|_{L^{\infty}} \frac{T^{\alpha-\beta}}{\Gamma(\alpha-\beta+1)}+\frac{\left|v_{2}\right| T^{1-\beta}}{\Gamma(2-\beta)} \\
+\frac{(\varphi(r)+\psi(r))\|m\|_{L^{\infty}} T^{1-\beta}}{\left|v_{1}\right| \Gamma(2-\beta)}\left(\frac{\left|a_{2}\right| T^{\alpha}}{\Gamma(\alpha+1)}+\frac{\left|b_{2}\right| T^{\alpha-\gamma}}{\Gamma(\alpha-\gamma+1)}\right) .
\end{gathered}
$$

Hence we obtain

$$
\begin{aligned}
\|h\| \leq & \left|v_{2}\right| T+\frac{\left|c_{1}\right|}{\left|a_{1}\right|}+\frac{\left|v_{2}\right| T^{1-\beta}}{\Gamma(2-\beta)}+(\varphi(r)+\psi(r))\|m\|_{L^{\infty}} \\
& \times\left(\frac{T^{\alpha-\beta}}{\Gamma(\alpha-\beta+1)}+\frac{T^{\alpha}}{\Gamma(\alpha+1)}+\left(\frac{T}{\left|v_{1}\right|}+\frac{T^{1-\beta}}{\left|v_{1}\right| \Gamma(2-\beta)}\right)\left(\frac{\left|a_{2}\right| T^{\alpha}}{\Gamma(\alpha+1)}+\frac{\left|b_{2}\right| T^{\alpha-\gamma}}{\Gamma(\alpha-\gamma+1)}\right)\right) \\
\leq & P+(\varphi(r)+\psi(r))\|m\|_{L^{\infty}} Q=l \quad \text { (a constant). }
\end{aligned}
$$

Step 3 ( $N$ maps bounded sets into equicontinuous sets in $\mathcal{X}$ ). Let $B_{r}$ be a bounded set of $\mathcal{X}$ as in Step 2. Let $0 \leq t_{1}<t_{2} \leq T$ and $x \in B_{r}$. For each $h \in N(x)$, then there is $u \in S_{F, x}$ such that $h(t)=(S u)(t)$. Since 


$$
\begin{aligned}
\left|\left(S_{1} u\right)\left(t_{2}\right)-\left(S_{1} u\right)\left(t_{1}\right)\right| & =\left|\int_{t_{1}}^{t_{2}} \frac{\left(t_{2}-s\right)^{\alpha-1}}{\Gamma(\alpha)} u(s) d s+\int_{0}^{t_{1}} \frac{\left(t_{2}-s\right)^{\alpha-1}-\left(t_{1}-s\right)^{\alpha-1}}{\Gamma(\alpha)} u(s) d s\right| \\
\leq & \frac{(\varphi(r)+\psi(r))\|m\|_{L^{\infty}}}{\Gamma(\alpha+1)}\left(\left(t_{2}-t_{1}\right)^{\alpha}+\left|t_{2}^{\alpha}-\left(t_{2}-t_{1}\right)^{\alpha}-t_{1}^{\alpha}\right|\right) \\
\leq & \frac{(\varphi(r)+\psi(r))\|m\|_{L^{\infty}}\left(t_{2}^{\alpha}-t_{1}^{\alpha}\right)}{\Gamma(\alpha+1)}, \\
\left|\left(S_{2} u\right)\left(t_{2}\right)-\left(S_{2} u\right)\left(t_{1}\right)\right|= & \left|-k_{u} t_{2}+\frac{c_{1}}{a_{1}}+k_{u} t_{1}-\frac{c_{1}}{a_{1}}\right| \\
\leq & \left(\frac{(\varphi(r)+\psi(r))\|m\|_{L^{\infty}}}{\left|v_{1}\right|}\left(\frac{\left|a_{2}\right| T^{\alpha}}{\Gamma(\alpha+1)}+\frac{\left|b_{2}\right| T^{\alpha-\gamma}}{\Gamma(\alpha-\gamma+1)}\right)+\left|v_{2}\right|\right)\left(t_{2}-t_{1}\right), \\
\left|{ }^{c} D^{\beta} h\left(t_{2}\right)-{ }^{c} D^{\beta} h\left(t_{1}\right)\right|= & \left|I^{\alpha-\beta} u\left(t_{2}\right)-\frac{k_{u} t_{2}^{1-\beta}}{\Gamma(2-\beta)}-I^{\alpha-\beta} u\left(t_{1}\right)+\frac{k_{u} t_{1}^{1-\beta} \mid}{\Gamma(2-\beta)}\right| \\
\leq & \left(\frac{(\varphi(r)+\psi(r))\|m\|_{L^{\infty}}}{\left|v_{1}\right| \Gamma(2-\beta)}\left(\frac{\left|a_{2}\right| T^{\alpha}}{\Gamma(\alpha+1)}+\frac{\left|b_{2}\right| T^{\alpha-\gamma}}{\Gamma(\alpha-\gamma+1)}\right)\right. \\
& \left.\quad+\frac{\left|v_{2}\right|}{\Gamma(2-\beta)}\right)\left(t_{2}^{1-\beta}-t_{1}^{1-\beta}\right)+\frac{(\varphi(r)+\psi(r))\|m\|_{L^{\infty}}\left(t_{2}^{\alpha-\beta}-t_{1}^{\alpha-\beta}\right)}{\Gamma(\alpha-\beta+1)},
\end{aligned}
$$

we obtain that (since $\alpha>1, \alpha-\beta>0$ and $1-\beta \geq 0$ )

$$
\left|h\left(t_{2}\right)-h\left(t_{1}\right)\right| \longrightarrow 0, \quad\left|{ }^{c} D^{\beta} h\left(t_{2}\right)-{ }^{c} D^{\beta} h\left(t_{1}\right)\right| \longrightarrow 0 \quad \text { as } t_{2} \longrightarrow t_{1}
$$

and the limits are independent of $x \in B_{r}$ and $h \in N(x)$.

Step 4 ( $N$ has a closed graph). Let $x_{n} \rightarrow x_{*}, h_{n} \in N\left(x_{n}\right)$, and $h_{n} \rightarrow h_{*} ;$ we need to show $h_{*} \in N\left(x_{*}\right)$. Now $h_{n} \in N\left(x_{n}\right)$ implies that there exists $u_{n} \in S_{F, x_{n}}$ such that $h_{n}(t)=\left(S u_{n}\right)(t)$ for $t \in[0, T]$. Let us consider the continuous linear operator $\Gamma: L^{1}([0, T], \mathbb{R}) \rightarrow \chi x$ given by

$$
(\Gamma u)(t)=\int_{0}^{t} \frac{(t-s)^{\alpha-1}}{\Gamma(\alpha)} u(s) d s-\frac{t}{v_{1}}\left(a_{2} \int_{0}^{T} \frac{(T-s)^{\alpha-1}}{\Gamma(\alpha)} u(s) d s+b_{2} \int_{0}^{T} \frac{(T-s)^{\alpha-\gamma-1}}{\Gamma(\alpha-\gamma)} u(s) d s\right)
$$

and denote $w(t)=v_{2} t+c_{1} / a_{1}$. Then $h_{n}(t)-w(t)=\left(\Gamma u_{n}\right)(t)$ and

$$
\begin{aligned}
\left\|h_{n}-h_{*}\right\|= & \max _{t \in[0, T]}\left|h_{n}(t)-w(t)-\left(h_{*}(t)-w(t)\right)\right| \\
& +\max _{t \in[0, T]}\left|{ }^{c} D^{\beta}\left(h_{n}-w\right)(t)-{ }^{c} D^{\beta}\left(h_{*}-w\right)(t)\right| \longrightarrow 0 \quad \text { as } n \longrightarrow \infty .
\end{aligned}
$$


We apply Lemma 2.3 to find that $\Gamma \circ S_{F}$ has closed graph and from the definition of $\Gamma$ we get $h_{n}-w \in \Gamma \circ S_{F}\left(x_{n}\right)$. Since $x_{n} \rightarrow x_{*}, h_{n}-w \rightarrow h_{*}-w$, it follows the existence of $u_{*} \in S_{F, x_{*}}$ such that $h_{*}-w=\Gamma\left(u_{*}\right)$. This means that $h_{*} \in N\left(x_{*}\right)$.

Step 5 (a priori bounds on solutions). Let $x \in \lambda N(x)$ for some $\lambda \in(0,1)$. Then there exists $u \in S_{F, x}$ such that $x(t)=\lambda(S u)(t)$ for $t \in[0, T]$. With the same arguments as in Step 2 of our proof, for each $t \in[0, T]$, we obtain

$$
|x(t)|+\left|{ }^{c} D^{\beta} x(t)\right| \leq P+(\varphi(\|x\|)+\psi(\|x\|))\|m\|_{L^{\infty}} Q .
$$

Thus

$$
\|x\| \leq P+(\varphi(\|x\|)+\psi(\|x\|))\|m\|_{L^{\infty}} Q .
$$

Now we set

$$
U=\{x \in \mathcal{X}:\|x\|<L\}
$$

Clearly, $U$ is an open subset of $\mathcal{X}$ and $0 \in U$. As a consequence of Steps $1-4$, together with the Arzela-Ascoli theorem, we can conclude that $N: \bar{U} \rightarrow P_{c p, c}(\mathcal{X})$ is upper semicontinuous and completely continuous. From the choice of the $U$, there is no $x \in \partial U$ such that $x \in$ $\lambda N(x)$ for some $\lambda \in(0,1)$. Therefore, by the nonlinear alternative of Leary-Schauder type (Theorem 2.11), we deduce that $N$ has a fixed point $x \in \bar{U}$, which is a solution of the problem (1.4), (1.5). This completes the proof.

Theorem 3.2. Assume that (H1) is satisfied and there exists $L_{1}>0$ such that

$$
\frac{L_{1}}{P_{1}+\left(\varphi\left(L_{1}\right)+\psi\left(L_{1}\right)\right)\|m\|_{L^{\infty}} Q_{1}}>1
$$

where

$$
\begin{gathered}
P_{1}=\frac{T^{\gamma} \Gamma(2-\gamma)\left|c_{2}\right|}{\left|b_{2}\right|}\left(1+\frac{T^{-\beta}}{\Gamma(2-\beta)}\right)+\left|\frac{b_{1} c_{2} T^{\gamma} \Gamma(2-\gamma)}{\left(a_{1}+b_{1}\right) b_{2}}-\frac{c_{1}}{a_{1}+b_{1}}\right| \\
Q_{1}=T^{\alpha}\left(1+\frac{\left|b_{1}\right|}{\left|a_{1}+b_{1}\right|}\right)\left(\frac{1}{\Gamma(\alpha+1)}+\frac{\Gamma(2-\gamma)}{\Gamma(\alpha-\gamma+1)}\right) \\
+T^{\alpha-\beta}\left(\frac{1}{\Gamma(\alpha-\beta+1)}+\frac{\Gamma(2-\gamma)}{\Gamma(2-\beta) \Gamma(\alpha-\gamma+1)}\right) .
\end{gathered}
$$

Then the problem (1.4), (1.6) has at least one solution on $[0, T]$.

Proof. To obtain the result, the main aim is to study the properties of the operator $M$ defined in (3.3). The proof of them is similar to those of Theorem 3.1, so we omit the details. Here 
we just give some estimations, which are needed in the following theorems. Let $x \in \mathcal{X}$ and $h \in M(x)$; then there exists $u \in S_{F, x}$ such that

$$
h(t)=(K u)(t), \quad \text { for } t \in[0, T]
$$

We put $K u=K_{1} u+K_{2} u$ and

$$
\left(K_{1} u\right)(t)=\int_{0}^{t} \frac{(t-s)^{\alpha-1}}{\Gamma(\alpha)} u(s) d s, \quad\left(K_{2} u\right)(t)=-k_{2}^{u} t-k_{1}^{u}
$$

here $k_{1}^{u}$ and $k_{2}^{u}$ are constants given by

$$
\begin{gathered}
k_{1}^{u}=\frac{b_{1} c_{2} T^{\gamma} \Gamma(2-\gamma)}{\left(a_{1}+b_{1}\right) b_{2}}-\frac{c_{1}}{a_{1}+b_{1}} \\
+\frac{b_{1}}{a_{1}+b_{1}}\left(\int_{0}^{T} \frac{(T-s)^{\alpha-1}}{\Gamma(\alpha)} u(s) d s-T^{\gamma} \Gamma(2-\gamma) \int_{0}^{T} \frac{(T-s)^{\alpha-\gamma-1}}{\Gamma(\alpha-\gamma)} u(s) d s\right), \\
k_{2}^{u}=\frac{\Gamma(2-\gamma)}{T^{1-\gamma}}\left(\int_{0}^{T} \frac{(T-s)^{\alpha-\gamma-1}}{\Gamma(\alpha-\gamma)} u(s) d s-\frac{c_{2}}{b_{2}}\right) .
\end{gathered}
$$

By simple calculations, we have

$$
\begin{gathered}
\left|\left(K_{1} u\right)(t)\right| \leq \int_{0}^{t} \frac{(t-s)^{\alpha-1}}{\Gamma(\alpha)}|u(s)| d s \leq(\varphi(\|x\|)+\psi(\|x\|))\|m\|_{L^{\infty}} \frac{T^{\alpha}}{\Gamma(\alpha+1)}, \\
\left|\left(K_{2} u\right)(t)\right| \leq T\left|k_{2}^{u}\right|+\left|k_{1}^{u}\right|, \\
T\left|k_{2}^{u}\right| \leq T^{\gamma} \Gamma(2-\gamma)\left((\varphi(\|x\|)+\psi(\|x\|))\|m\|_{L^{\infty}} \frac{T^{\alpha-\gamma}}{\Gamma(\alpha-\gamma+1)}+\frac{\left|c_{2}\right|}{\left|b_{2}\right|}\right), \\
\left|k_{1}^{u}\right| \leq \frac{\left|b_{1}\right|}{\left|a_{1}+b_{1}\right|}\left((\varphi(\|x\|)+\psi(\|x\|))\|m\|_{L^{\infty}} \frac{T^{\alpha}}{\Gamma(\alpha+1)}+(\varphi(\|x\|)+\psi(\|x\|))\|m\|_{L^{\infty}} \frac{\Gamma(2-\gamma) T^{\alpha}}{\Gamma(\alpha-\gamma+1)}\right) \\
+\left|\frac{b_{1} c_{2} T^{\gamma} \Gamma(2-\gamma)}{\left(a_{1}+b_{1}\right) b_{2}}-\frac{c_{1}}{a_{1}+b_{1} \mid}\right|, \\
\left|{ }^{c} D^{\beta} h(t)\right| \leq \int_{0}^{t} \frac{(t-s)^{\alpha-\beta-1}}{\Gamma(\alpha-\beta)}|u(s)| d s+\frac{\left|k_{2}^{u}\right| T^{1-\beta}}{\Gamma(2-\beta)} \\
\leq(\varphi(\|x\|)+\psi(\|x\|))\|m\|_{L^{\infty}} \frac{T^{\alpha-\beta}}{\Gamma(\alpha-\beta+1)}+\frac{\Gamma(2-\gamma) T^{\gamma-\beta}}{\Gamma(2-\beta)} \\
\times\left((\varphi(\|x\|)+\psi(\|x\|))\|m\|_{L^{\infty}} \frac{T^{\alpha-\gamma}}{\Gamma(\alpha-\gamma+1)}+\frac{\left|c_{2}\right|}{\left|b_{2}\right|}\right) .
\end{gathered}
$$


Hence we obtain

$$
\begin{aligned}
\|h\| \leq & \frac{T^{\gamma} \Gamma(2-\gamma)\left|c_{2}\right|}{\left|b_{2}\right|}\left(1+\frac{T^{-\beta}}{\Gamma(2-\beta)}\right)+\left|\frac{b_{1} c_{2} T^{\gamma} \Gamma(2-\gamma)}{\left(a_{1}+b_{1}\right) b_{2}}-\frac{c_{1}}{a_{1}+b_{1}}\right| \\
& +(\varphi(\|x\|)+\psi(\|x\|))\|m\|_{L^{\infty}} T^{\alpha}\left(1+\frac{\left|b_{1}\right|}{\left|a_{1}+b_{1}\right|}\right)\left(\frac{1}{\Gamma(\alpha+1)}+\frac{\Gamma(2-\gamma)}{\Gamma(\alpha-\gamma+1)}\right) \\
& +(\varphi(\|x\|)+\psi(\|x\|))\|m\|_{L^{\infty}} T^{\alpha-\beta}\left(\frac{1}{\Gamma(\alpha-\beta+1)}+\frac{\Gamma(2-\gamma)}{\Gamma(2-\beta) \Gamma(\alpha-\gamma+1)}\right) .
\end{aligned}
$$

This is the end of the proof.

\subsection{Nonconvex Case}

Now we study the case when $F$ is not necessarily convex valued.

A subset $A$ of $L^{1}([0, T], \mathbb{R})$ is decomposable if for all $u, v \in A$ and $J \subseteq[0, T]$ Lebesgue measurable, then $u_{X J}+v_{X[0, T]-J} \in A$, where $X$ stands for the characteristic function.

$(\mathrm{H} 2): F:[0, T] \times \mathbb{R} \times \mathbb{R} \rightarrow P_{\text {cp }}(\mathbb{R})$ is a multivalued map such that (1) $(t, x, y) \rightarrow F(t, x, y)$ is $\Sigma \otimes \mathbb{B}_{\mathbb{R}} \otimes \mathbb{B}_{\mathbb{R}}$ measurable; (2) $(x, y) \rightarrow F(t, x, y)$ is lower semicontinuous for a.e. $t \in[0, T]$.

Theorem 3.3. Let (H1)(2), (H2), and relation (3.6) hold; then the problem (1.4), (1.5) has at least one solution on $[0, T]$.

Proof. From (H1)(2), (H2), and [33, Lemma 4.1], the map

$$
\mathcal{F}: x \rightarrow P\left(L^{1}([0, T], \mathbb{R})\right), \quad x \longrightarrow \mathcal{F}(x)=S_{F, x}
$$

is lower semicontinuous and has nonempty closed and decomposable values. Then from a selection theorem due to Bressan and Colombo [34], there exists a continuous function $f$ : $x \rightarrow L^{1}([0, T], \mathbb{R})$ such that $f(x) \in \mathcal{F}(x)$ for all $x \in \mathcal{X}$. That is to say, we have $f(x)(t) \in$ $F\left(t, x(t),{ }^{c} D^{\beta} x(t)\right)$ for a.e. $t \in[0, T]$. Now consider the problem

$$
{ }^{c} D^{\alpha} x(t)=f(x)(t), \quad t \in[0, T]
$$

with the boundary conditions (1.5). Note that if $x \in \mathcal{X}$ is a solution of the problem (3.29), then $x$ is a solution to the problem (1.4), (1.5).

Problem (3.29) is then reformulated as a fixed point problem for the operator $N_{1}$ : $x \rightarrow x$ defined by

$$
N_{1}(x)(t)=(S f(x))(t)
$$

It can easily be shown that $N_{1}$ is continuous and completely continuous and satisfies all conditions of the Leray-Schauder nonlinear alternative for single-valued maps [31]. The 
remaining part of the proof is similar to that of Theorem 3.1, so we omit it. This completes the proof.

Theorem 3.4. Let (H1)(2), (H2), and relation (3.21) hold, then the problem (1.4), (1.6) has at least one solution on $[0, T]$.

The proof of this theorem is similar to that of Theorem 3.3.

(H3): $F:[0, T] \times \mathbb{R} \times \mathbb{R} \rightarrow P_{c p}(\mathbb{R})$ is a multivalued map such that (1) $F$ is integrably bounded and the map $t \rightarrow F(t, x, y)$ is measurable for all $x, y \in \mathbb{R}$; (2) there exists $m \in L^{\infty}\left([0, T], \mathbb{R}^{+}\right)$such that for a.e. $t \in[0, T]$ and all $x_{1}, x_{2}, y_{1}, y_{2} \in \mathbb{R}$,

$$
h\left(F\left(t, x_{1}, y_{1}\right), F\left(t, x_{2}, y_{2}\right)\right) \leq m(t)\left(\left|x_{1}-x_{2}\right|+\left|y_{1}-y_{2}\right|\right) \text {. }
$$

Theorem 3.5. Let (H3) hold, if, in addition,

$$
\|m\|_{L^{\infty}}\left[\frac{T^{\alpha}}{\Gamma(\alpha+1)}+\frac{T^{\alpha-\beta}}{\Gamma(\alpha-\beta+1)}+\left(\frac{T}{\left|v_{1}\right|}+\frac{T^{1-\beta}}{\left|v_{1}\right| \Gamma(2-\beta)}\right)\left(\frac{\left|a_{2}\right| T^{\alpha}}{\Gamma(\alpha+1)}+\frac{\left|b_{2}\right| T^{\alpha-\gamma}}{\Gamma(\alpha-\gamma+1)}\right)\right]<1,
$$

then the problem (1.4), (1.5) has at least one solution on $[0, T]$.

Proof. From (H3), we have that the multivalued map $t \rightarrow F\left(t, x(t),{ }^{c} D^{\beta} x(t)\right)$ is measurable [28, Proposition 2.7.9] and closed valued for each $x \in \mathcal{X}$. Hence it has measurable selection [28, Theorem 2.2.1] and the set $S_{F, x}$ is nonempty. Let $N$ be defined in (3.2). We will show that, under this situation, $N$ satisfies the requirements of Theorem 2.12.

Step 1. For each $x \in \mathcal{X}, N(x) \in P_{\mathrm{cl}}(\mathcal{X})$. Let $h_{n} \in N(x), n \geq 1$ such that $h_{n} \rightarrow h$ in $\mathcal{X}$. Then $h \in \mathcal{X}$ and there exists $u_{n} \in S_{F, x}, n \geq 1$ such that

$$
h_{n}(t)=\left(S u_{n}\right)(t), \quad t \in[0, T] .
$$

By (H3), the sequence $u_{n}$ is integrable bounded. Since $F$ has compact values, we may pass to a subsequence if necessary to get that $u_{n}$ converges to $u$ in $L^{1}([0, T], \mathbb{R})$. Thus $u \in S_{F, x}$ and for each $t \in[0, T]$

$$
h_{n}(t) \longrightarrow h(t)=(S u)(t)
$$

This means that $h \in N(x)$ and $N(x)$ is closed.

Step 2. There exists $\rho<1$ such that

$$
h(N(x), N(y)) \leq \rho\|x-y\|, \quad \forall x, y \in X .
$$

Let $x, y \in \mathcal{X}$ and $h_{1} \in N(y)$; then there exists $u_{1} \in S_{F, y}$ such that

$$
h_{1}(t)=\left(S u_{1}\right)(t), \quad t \in[0, T]
$$


From (H3)(2), we deduce

$$
\begin{aligned}
& h\left(F\left(t, x(t),{ }^{c} D^{\beta} x(t)\right), F\left(t, y(t),{ }^{c} D^{\beta} y(t)\right)\right) \\
& \quad \leq m(t)\left(|x(t)-y(t)|+\left|{ }^{c} D^{\beta} x(t)-{ }^{c} D^{\beta} y(t)\right|\right) .
\end{aligned}
$$

Hence, for a.e. $t \in[0, T]$, there exists $v \in F\left(t, x(t),{ }^{c} D^{\beta} x(t)\right)$ such that

$$
\left|u_{1}(t)-v\right| \leq m(t)\left(|x(t)-y(t)|+\left|{ }^{c} D^{\beta} x(t)-{ }^{c} D^{\beta} y(t)\right|\right)
$$

Consider the multivalued map $V:[0, T] \rightarrow P(\mathbb{R})$ given by

$$
V(t)=\left\{u \in \mathbb{R}:\left|u_{1}(t)-u\right| \leq m(t)\left(|x(t)-y(t)|+\left|{ }^{c} D^{\beta} x(t)-{ }^{c} D^{\beta} y(t)\right|\right)\right\} .
$$

Since $u_{1}(t), \alpha(t)=m(t)\left(|x(t)-y(t)|+\left|{ }^{c} D^{\beta} x(t)-{ }^{c} D^{\beta} y(t)\right|\right)$ are measurable, [35, Theorem III.41] implies that $V$ is measurable. It follows from (H3) that the map $t \rightarrow F\left(t, x(t),{ }^{c} D^{\beta} x(t)\right)$ is measurable. Hence by (3.38) and [28, Proposition 2.1.43], the multivalued map $t \rightarrow V(t) \cap$ $F\left(t, x(t),{ }^{c} D^{\beta} x(t)\right)$ is measurable and nonempty closed valued. Therefore, we can find $u_{2}(t) \in$ $F\left(t, x(t),{ }^{c} D^{\beta} x(t)\right)$ such that for a.e. $t \in[0, T]$,

$$
\left|u_{1}(t)-u_{2}(t)\right| \leq m(t)\left(|x(t)-y(t)|+\left|{ }^{c} D^{\beta} x(t)-{ }^{c} D^{\beta} y(t)\right|\right)
$$

Let $h_{2}(t)=\left(S u_{2}\right)(t)$, that is, $h_{2} \in N(x)$. Since

$$
\begin{aligned}
\left|\left(S_{1} u_{1}\right)(t)-\left(S_{1} u_{2}\right)(t)\right| & =\left|\int_{0}^{t} \frac{(t-s)^{\alpha-1}}{\Gamma(\alpha)}\left(u_{1}(s)-u_{2}(s)\right) d s\right| \\
& \leq \int_{0}^{t} \frac{(t-s)^{\alpha-1}}{\Gamma(\alpha)} m(s)\left(|x(s)-y(s)|+\left|{ }^{c} D^{\beta} x(s)-{ }^{c} D^{\beta} y(s)\right|\right) d s \\
& \leq \frac{\|m\|_{L^{\infty}} T^{\alpha}}{\Gamma(\alpha+1)}\|x-y\|, \\
\left|\left(S_{2} u_{1}\right)(t)-\left(S_{2} u_{2}\right)(t)\right| & =\left|t\left(k_{u_{1}}-k_{u_{2}}\right)\right| \\
& \leq T \mid \frac{a_{2}}{v_{1}} \int_{0}^{T} \frac{(T-s)^{\alpha-1}}{\Gamma(\alpha)}\left(u_{1}(s)-u_{2}(s)\right) d s \\
& +\frac{b_{2}}{v_{1}} \int_{0}^{T} \frac{(T-s)^{\alpha-\gamma-1}}{\Gamma(\alpha-\gamma)}\left(u_{1}(s)-u_{2}(s)\right) d s \mid \\
& \leq \frac{\mid m \|_{L^{\infty}} T}{\left|v_{1}\right|}\left(\frac{\left|a_{2}\right| T^{\alpha}}{\Gamma(\alpha+1)}+\frac{\left|b_{2}\right| T^{\alpha-\gamma}}{\Gamma(\alpha-\gamma+1)}\right)\|x-y\|
\end{aligned}
$$


Abstract and Applied Analysis

$$
\begin{aligned}
\left|{ }^{c} D^{\beta} h_{1}(t)-{ }^{c} D^{\beta} h_{2}(t)\right|= & \left|I^{\alpha-\beta} u_{1}(t)-\frac{k_{u_{1}} t^{1-\beta}}{\Gamma(2-\beta)}-I^{\alpha-\beta} u_{2}(t)+\frac{k_{u_{2}} t^{1-\beta}}{\Gamma(2-\beta)}\right| \\
\leq & \frac{\|m\|_{L^{\infty}} T^{\alpha-\beta}}{\Gamma(\alpha-\beta+1)}\|x-y\| \\
& +\frac{\|m\|_{L^{\infty}} T^{1-\beta}}{\left|v_{1}\right| \Gamma(2-\beta)}\left(\frac{\left|a_{2}\right| T^{\alpha}}{\Gamma(\alpha+1)}+\frac{\left|b_{2}\right| T^{\alpha-\gamma}}{\Gamma(\alpha-\gamma+1)}\right)\|x-y\|,
\end{aligned}
$$

we obtain

$$
\begin{aligned}
\left\|h_{1}-h_{2}\right\| \leq\|m\|_{L^{\infty}}[ & \frac{T^{\alpha}}{\Gamma(\alpha+1)}+\frac{T^{\alpha-\beta}}{\Gamma(\alpha-\beta+1)} \\
& \left.\quad\left(\frac{T}{\left|v_{1}\right|}+\frac{T^{1-\beta}}{\left|v_{1}\right| \Gamma(2-\beta)}\right)\left(\frac{\left|a_{2}\right| T^{\alpha}}{\Gamma(\alpha+1)}+\frac{\left|b_{2}\right| T^{\alpha-\gamma}}{\Gamma(\alpha-\gamma+1)}\right)\right]\|x-y\| .
\end{aligned}
$$

Denote

$$
\rho=\|m\|_{L^{\infty}}\left[\frac{T^{\alpha}}{\Gamma(\alpha+1)}+\frac{T^{\alpha-\beta}}{\Gamma(\alpha-\beta+1)}+\left(\frac{T}{\left|v_{1}\right|}+\frac{T^{1-\beta}}{\left|v_{1}\right| \Gamma(2-\beta)}\right)\left(\frac{\left|a_{2}\right| T^{\alpha}}{\Gamma(\alpha+1)}+\frac{\left|b_{2}\right| T^{\alpha-\gamma}}{\Gamma(\alpha-\gamma+1)}\right)\right]
$$

By using an analogous relation obtained by interchanging the roles of $x$ and $y$, we get

$$
h(N(x), N(y)) \leq \rho\|x-y\| .
$$

Therefore, from condition (3.32), Theorem 2.12 implies that $N$ has a fixed point, which is a solution of the problem (1.4), (1.5). This completes the proof.

Theorem 3.6. Let (H3) hold, if, in addition,

$$
\begin{aligned}
& \|m\|_{L^{\infty}} T^{\alpha}\left(1+\frac{\left|b_{1}\right|}{\left|a_{1}+b_{1}\right|}\right)\left(\frac{1}{\Gamma(\alpha+1)}+\frac{\Gamma(2-\gamma)}{\Gamma(\alpha-\gamma+1)}\right) \\
& +\|m\|_{L^{\infty}} T^{\alpha-\beta}\left(\frac{1}{\Gamma(\alpha-\beta+1)}+\frac{\Gamma(2-\gamma)}{\Gamma(2-\beta) \Gamma(\alpha-\gamma+1)}\right)<1,
\end{aligned}
$$

then the problem (1.4), (1.6) has at least one solution on $[0, T]$. 
Using the arguments employed in the proof of Theorem 3.5, we can prove this theorem similarly. Hence the details are omitted here.

\section{Integral Boundary Conditions}

In this section, the existence results of the problems (1.4), (1.5) and (1.4), (1.6) obtained in the previous section will be extended to the ones of the problems of fractional differential inclusions (1.4) subject to the integral boundary conditions (1.7) and (1.8).

Lemma 4.1. For any $y, \xi, x \in C([0, T], \mathbb{R})$, the unique solution of the fractional separated integral boundary value problem,

$$
\begin{gathered}
{ }^{c} D^{\alpha} x(t)=y(t), \quad t \in[0, T], 1<\alpha \leq 2, \\
a_{1} x(0)+b_{1}\left({ }^{c} D^{\gamma} x(0)\right)=c_{1} \int_{0}^{T} \xi(s) d s, \\
a_{2} x(T)+b_{2}\left({ }^{c} D^{\gamma} x(T)\right)=c_{2} \int_{0}^{T} x(s) d s, \quad 0<\gamma<1,
\end{gathered}
$$

is given by

$$
\begin{aligned}
x(t)= & \int_{0}^{t} \frac{(t-s)^{\alpha-1}}{\Gamma(\alpha)} y(s) d s \\
& -\frac{t}{v_{1}}\left(a_{2} \int_{0}^{T} \frac{(T-s)^{\alpha-1}}{\Gamma(\alpha)} y(s) d s+b_{2} \int_{0}^{T} \frac{(T-s)^{\alpha-\gamma-1}}{\Gamma(\alpha-\gamma)} y(s) d s\right) \\
& +\frac{c_{2} t}{v_{1}} \int_{0}^{T} x(s) d s+\frac{c_{1}\left(v_{1}-a_{2} t\right)}{a_{1} v_{1}} \int_{0}^{T} \xi(s) d s .
\end{aligned}
$$

Lemma 4.2. For any $y, \xi, x \in C([0, T], \mathbb{R})$, the unique solution of the fractional nonseparated integral boundary value problem,

$$
\begin{gathered}
{ }^{c} D^{\alpha} x(t)=y(t), \quad t \in[0, T], 1<\alpha \leq 2, \\
a_{1} x(0)+b_{1} x(T)=c_{1} \int_{0}^{T} \xi(s) d s, \\
a_{2}\left({ }^{c} D^{\gamma} x(0)\right)+b_{2}\left({ }^{c} D^{\gamma} x(T)\right)=c_{2} \int_{0}^{T} x(s) d s, \quad 0<\gamma<1,
\end{gathered}
$$


is given by

$$
\begin{aligned}
x(t)= & \int_{0}^{t} \frac{(t-s)^{\alpha-1}}{\Gamma(\alpha)} y(s) d s-\frac{t \Gamma(2-\gamma)}{T^{1-\gamma}} \int_{0}^{T} \frac{(T-s)^{\alpha-\gamma-1}}{\Gamma(\alpha-\gamma)} y(s) d s+\frac{t \Gamma(2-\gamma) c_{2}}{T^{1-\gamma} b_{2}} \int_{0}^{T} x(s) d s \\
& -\frac{b_{1}}{a_{1}+b_{1}}\left(\int_{0}^{T} \frac{(T-s)^{\alpha-1}}{\Gamma(\alpha)} y(s) d s-T^{\gamma} \Gamma(2-\gamma) \int_{0}^{T} \frac{(T-s)^{\alpha-\gamma-1}}{\Gamma(\alpha-\gamma)} y(s) d s\right) \\
& -\frac{b_{1} T^{\gamma} \Gamma(2-\gamma) c_{2}}{b_{2}\left(a_{1}+b_{1}\right)} \int_{0}^{T} x(s) d s+\frac{c_{1}}{a_{1}+b_{1}} \int_{0}^{T} \xi(s) d s .
\end{aligned}
$$

To obtain the existence results of the problems (1.4), (1.7) and (1.4), (1.8), in view of Lemmas 4.1 and 4.2, we define two operators $\Pi, \Omega: \mathcal{X} \rightarrow P(\mathcal{X})$ as

$$
\begin{aligned}
& \Pi(x)=\left\{h \in \mathcal{X}: h=H v, v \in S_{F, x}\right\}, \\
& \Omega(x)=\left\{h \in \mathcal{X}: h=Z v, v \in S_{F, x}\right\}
\end{aligned}
$$

with

$$
\begin{aligned}
(H v)(t)= & \int_{0}^{t} \frac{(t-s)^{\alpha-1}}{\Gamma(\alpha)} v(s) d s \\
& -\frac{t}{v_{1}}\left(a_{2} \int_{0}^{T} \frac{(T-s)^{\alpha-1}}{\Gamma(\alpha)} v(s) d s+b_{2} \int_{0}^{T} \frac{(T-s)^{\alpha-\gamma-1}}{\Gamma(\alpha-\gamma)} v(s) d s\right) \\
& +\frac{c_{2} t}{v_{1}} \int_{0}^{T} h(s, x(s)) d s+\frac{c_{1}}{a_{1}} \int_{0}^{T} g(s, x(s)) d s-\frac{c_{1} a_{2} t}{a_{1} v_{1}} \int_{0}^{T} g(s, x(s)) d s, \\
(Z v)(t)= & \int_{0}^{t} \frac{(t-s)^{\alpha-1}}{\Gamma(\alpha)} v(s) d s-\frac{t \Gamma(2-\gamma)}{T^{1-\gamma}} \int_{0}^{T} \frac{(T-s)^{\alpha-\gamma-1}}{\Gamma(\alpha-\gamma)} v(s) d s \\
& +\frac{t \Gamma(2-\gamma) c_{2}}{T^{1-\gamma} b_{2}} \int_{0}^{T} h(s, x(s)) d s \\
& -\frac{b_{1}}{a_{1}+b_{1}}\left(\int_{0}^{T} \frac{(T-s)^{\alpha-1}}{\Gamma(\alpha)} v(s) d s-T^{\gamma} \Gamma(2-\gamma) \int_{0}^{T} \frac{(T-s)^{\alpha-\gamma-1}}{\Gamma(\alpha-\gamma)} v(s) d s\right) \\
& -\frac{b_{1} T^{\gamma} \Gamma(2-\gamma) c_{2}}{b_{2}\left(a_{1}+b_{1}\right)} \int_{0}^{T} h(s, x(s)) d s+\frac{c_{1}}{a_{1}+b_{1}} \int_{0}^{T} g(s, x(s)) d s .
\end{aligned}
$$

Observe that if $x \in \mathcal{X}$ is a fixed point of the operator $\Pi$ (the operator $\Omega$ ), that is, $x \in \Pi(x)$ $(x \in \Omega(x))$, then $x$ is a solution of the problem (1.4), (1.7) (the problem (1.4), (1.8)).

From the definitions of the operators $N$, $\Pi$ (see (3.2), (4.5)), we know that the difference between them is very apparent, that is, $c_{1}, c_{2}$ in (3.2) were replaced by $c_{1} \int_{0}^{T} g(s, x(s)) d s$ and $c_{2} \int_{0}^{T} h(s, x(s)) d s$ in (4.5). This fact is also true for the operators $M$, $\Omega$ (see (3.3), (4.6)). 
In the following, we state some existence results for the problems (1.4), (1.7) and (1.4), (1.8). We omit the proofs as these are similar to the ones given in Section 3.

(A1): The functions $g, h:[0, T] \times \mathbb{R} \rightarrow \mathbb{R}$ are continuous. There exist functions $m_{2}, m_{3} \in$ $L^{1}\left([0, T], \mathbb{R}^{+}\right)$and $\varphi_{2}, \varphi_{3}:[0, \infty) \rightarrow(0, \infty)$ continuous, nondecreasing such that

$$
|g(t, x)| \leq m_{2}(t) \varphi_{2}(|x|), \quad|h(t, x)| \leq m_{3}(t) \varphi_{3}(|x|)
$$

for all $x \in \mathbb{R}$ and a.e. $t \in[0, T]$.

Theorem 4.3. Assume that (H1) and (A1) hold. If there exists a constant $I>0$ such that

$$
\frac{I}{(\varphi(I)+\psi(I))\|m\|_{L^{\infty}} Q+\varphi_{3}(I)\left\|m_{3}\right\|_{L^{1}} R+\varphi_{2}(I)\left\|m_{2}\right\|_{L^{1}} W}>1
$$

here $Q$ is defined by (3.7) and

$$
R=\frac{\left|c_{2}\right| T}{\left|v_{1}\right|}\left(1+\frac{T^{-\beta}}{\Gamma(2-\beta)}\right), \quad W=\frac{\left|c_{1}\right|}{\left|a_{1}\right|}\left(1+\frac{\left|a_{2}\right| T}{\left|v_{1}\right|}\left(1+\frac{T^{-\beta}}{\Gamma(2-\beta)}\right)\right) .
$$

Then the boundary value problem (1.4), (1.7) has at least one solution on $[0, T]$.

Theorem 4.4. Assume that (H1) and (A1) hold. If there exists a constant $I_{1}>0$ such that

$$
\frac{I_{1}}{\left(\varphi\left(I_{1}\right)+\psi\left(I_{1}\right)\right)\|m\|_{L^{\infty}} Q_{1}+\varphi_{3}\left(I_{1}\right)\left\|m_{3}\right\|_{L^{1}} R_{1}+\varphi_{2}\left(I_{1}\right)\left\|m_{2}\right\|_{L^{1}} W_{1}}>1,
$$

here $Q_{1}$ is defined by (3.22) and

$$
R_{1}=\frac{\left|c_{2}\right| T^{\gamma} \Gamma(2-\gamma)}{\left|b_{2}\right|}\left(1+\frac{\left|b_{1}\right|}{\left|a_{1}+b_{1}\right|}+\frac{T^{-\beta}}{\Gamma(2-\beta)}\right), \quad W_{1}=\frac{\left|c_{1}\right|}{\left|a_{1}+b_{1}\right|} .
$$

Then the boundary value problem (1.4), (1.8) has at least one solution on $[0, T]$.

Theorem 4.5. Assume that (H1)(2), (H2), (A1) and condition (4.9) hold. Then the boundary value problem (1.4), (1.7) has at least one solution on $[0, T]$.

Theorem 4.6. Assume that (H1)(2), (H2), (A1) and condition (4.11) hold. Then the boundary value problem (1.4), (1.8) has at least one solution on $[0, T]$.

(A2): The functions $g, h:[0, T] \times \mathbb{R} \rightarrow \mathbb{R}$ are continuous and satisfy

$$
|g(t, x)-g(t, y)| \leq m_{2}(t)|x-y|, \quad|h(t, x)-h(t, y)| \leq m_{3}(t)|x-y|
$$

for all $x, y \in \mathbb{R}$ and a.e. $t \in[0, T]$; here $m_{2}, m_{3} \in L^{1}\left([0, T], \mathbb{R}^{+}\right)$. 
Theorem 4.7. Assume that (H3) and (A2) hold. If, in addition,

$$
\|m\|_{L^{\infty}} Q+\left\|m_{3}\right\|_{L^{1}} R+\left\|m_{2}\right\|_{L^{1}} W<1,
$$

here $Q$ is defined by (3.7) and $R, W$ are defined by (4.10), then the boundary value problem (1.4), (1.7) has at least one solution on $[0, T]$.

Theorem 4.8. Assume that (H3) and (A2) hold. If, in addition,

$$
\|m\|_{L^{\infty}} Q_{1}+\left\|m_{3}\right\|_{L^{1}} R_{1}+\left\|m_{2}\right\|_{L^{1}} W_{1}<1
$$

here $Q_{1}$ is defined by (3.22) and $R_{1}, W_{1}$ are defined by (4.12), then the boundary value problem (1.4), (1.8) has at least one solution on $[0, T]$.

\section{Examples}

In this section, we give two simple examples to show the applicability of our results.

Example 5.1. Consider the following fractional boundary value problem:

$$
\begin{gathered}
{ }^{c} D^{3 / 2} x(t) \in F\left(t, x(t),{ }^{c} D^{3 / 4} x(t)\right), \quad t \in[0,1], \\
x(0)-\frac{1}{2}\left({ }^{c} D^{1 / 2} x(0)\right)=2.5 \\
2 x(1)+\frac{1}{3}\left({ }^{c} D^{1 / 2} x(1)\right)=-\frac{1}{3}
\end{gathered}
$$

where $\alpha=3 / 2, \beta=3 / 4, \gamma=1 / 2, a_{1}=1, b_{1}=-1 / 2, c_{1}=2.5, a_{2}=2, b_{2}=1 / 3, c_{2}=-1 / 3, T=1$, and $F:[0,1] \times \mathbb{R} \times \mathbb{R} \rightarrow P(\mathbb{R})$ is a multivalued map given by

$$
F(t, x, y)=\left\{u \in \mathbb{R}: e^{-|x|}-\frac{|y|}{1+|y|}+\sin t \leq u \leq 5+\frac{|x|}{1+x^{2}}+6 t^{3}+\cos y\right\} .
$$

In the context of this problem, we have

$$
\|F(t, x, y)\|=\sup \{|v|: v \in F(t, x, y)\} \leq 7+6 t^{3} \leq 13, \quad \text { for } t \in[0,1], x, y \in \mathbb{R}
$$

It is clear that $F$ is convex compact valued and is of Carathéodory type. Let $m(t) \equiv 1$ and $\varphi(|x|) \equiv 3, \psi(|y|) \equiv 10$; we get for $t \in[0,1], x, y \in \mathbb{R}$

$$
\|F(t, x, y)\|=\sup \{|v|: v \in F(t, x, y)\} \leq m(t)(\varphi(|x|)+\psi(|y|)) .
$$


As for the condition (3.6), since $P+(\varphi(|x|)+\psi(|y|))\|m\|_{L^{\infty}} Q=P+13 Q(P, Q$ defined in (3.6)) is a constant, we can choose $L$ large enough so that

$$
\frac{L}{P+(\varphi(L)+\psi(L))\|m\|_{L^{\infty}} Q}>1
$$

Thus, by the conclusion of Theorem 3.1, the boundary value problem (5.1) has at least one solution on $[0,1]$.

Example 5.2. Consider the following fractional differential inclusion with integral boundary conditions:

$$
\begin{gathered}
{ }^{c} D^{7 / 4} x(t) \in F\left(t, x(t),{ }^{c} D^{1 / 2} x(t)\right), \quad t \in[0,1], \\
3 x(0)+\frac{1}{3} x(1)=\int_{0}^{1} g(s, x(s)) d s, \\
2\left({ }^{c} D^{1 / 4} x(0)\right)+3\left({ }^{c} D^{1 / 4} x(1)\right)=\frac{1}{4} \int_{0}^{1} h(s, x(s)) d s,
\end{gathered}
$$

where $\alpha=7 / 4, \beta=1 / 2, \gamma=1 / 4, T=1, a_{1}=3, b_{1}=1 / 3, c_{1}=1, a_{2}=2, b_{2}=3, c_{2}=1 / 4$,

$$
\begin{gathered}
F(t, x, y)=\left[-l_{1}(t)-\frac{\sin x}{(4+t)^{2}}-2,-\frac{1}{10}\right] \bigcup\left[0, \frac{1}{16} \frac{|y|}{1+|y|}+l_{2}(t)\right], \\
g(t, x)=\frac{1}{(3+t)^{2}} \cos x, \quad h(t, x)=x,
\end{gathered}
$$

and $l_{1}, l_{2} \in L^{1}\left([0,1], \mathbb{R}^{+}\right)$.

From the data given above, we have for $t \in[0,1], x, y \in \mathbb{R}$,

$$
\begin{gathered}
\sup \{|u|: u \in F(t, x, y)\} \leq 3+\frac{1}{(4+t)^{2}}+l_{1}(t)+l_{2}(t), \\
h\left(F\left(t, x_{1}, y_{1}\right), F\left(t, x_{2}, y_{2}\right)\right) \leq \frac{1}{(4+t)^{2}}\left|x_{1}-x_{2}\right|+\frac{1}{16}\left|y_{1}-y_{2}\right|, \\
|g(t, x)-g(t, y)| \leq \frac{1}{(3+t)^{2}}|x-y|, \quad|h(t, x)-h(t, y)| \leq|x-y| .
\end{gathered}
$$

Then let $m_{2}(t)=1 /(3+t)^{2}, m_{3}(t)=1$, and $m(t)=1 / 16+1 /(4+t)^{2}$; we have

$$
\begin{gathered}
h\left(F\left(t, x_{1}, y_{1}\right), F\left(t, x_{2}, y_{2}\right)\right) \leq m(t)\left(\left|x_{1}-x_{2}\right|+\left|y_{1}-y_{2}\right|\right) \\
\|m\|_{L^{\infty}} Q_{1}+\left\|m_{3}\right\|_{L^{1}} R_{1}+\left\|m_{2}\right\|_{L^{1}} W_{1} \leq \frac{1}{8} \times 3.1071+1 \times 0.1707+\frac{1}{9} \times \frac{3}{10}=0.5924<1 .
\end{gathered}
$$


Here $Q_{1}$ is defined by (3.22) and $R_{1}, W_{1}$ are defined by (4.12). Hence all the assumptions of Theorem 4.8 are satisfied, and by the conclusion of it, the boundary value problem (5.6) has at least one solution on $[0,1]$.

\section{Acknowledgment}

This project was supported by NNSF of China Grants nos. 11271087 and 61263006.

\section{References}

[1] D. Baleanu, J. A. T. Machado, and A. C. J. Luo, Fractional Dynamics and Control, Springer, New York, NY, USA, 2012.

[2] J. Sabatier, O. P. Agrawal, and J. A. T. Machado, Eds., Advances in Fractional Calculus: Theoretical Developments and Applications in Physics and Engineering, Springer, Dordrecht, The Netherlands, 2007.

[3] V. Lakshmikantham, S. Leela, and J. Vasundhara Devi, Theory of Fractional Dynamic Systems, Cambridge Scientific, 2009.

[4] B. Ahmad and J. J. Nieto, "Anti-periodic fractional boundary value problems with nonlinear term depending on lower order derivative," Fractional Calculus and Applied Analysis, vol. 15, no. 3, pp. 451462, 2012.

[5] B. Ahmad and J. J. Nieto, "Anti-periodic fractional boundary value problems," Computers $\mathcal{E}$ Mathematics with Applications, vol. 62, no. 3, pp. 1150-1156, 2011.

[6] B. Ahmad and S. K. Ntouyas, "A note on fractional differential equations with fractional separated boundary conditions," Abstract and Applied Analysis, vol. 2012, Article ID 818703, 11 pages, 2012.

[7] Z. Bai, "On positive solutions of a nonlocal fractional boundary value problem," Nonlinear Analysis. Theory, Methods E Applications, vol. 72, no. 2, pp. 916-924, 2010.

[8] D. Băleanu, O. G. Mustafa, and R. P. Agarwal, "An existence result for a superlinear fractional differential equation," Applied Mathematics Letters, vol. 23, no. 9, pp. 1129-1132, 2010.

[9] A. Chen and Y. Chen, "Existence of solutions to anti-periodic boundary value problem for nonlinear fractional differential equations," Differential Equations and Dynamical Systems, vol. 19, no. 3, pp. 237252, 2011.

[10] A. Chen and Y. Tian, "Existence of three positive solutions to three-point boundary value problem of nonlinear fractional differential equation," Differential Equations and Dynamical Systems, vol. 18, no. 3, pp. 327-339, 2010.

[11] R. A. Khan, M. U. Rehman, and J. Henderson, “Existence and uniqueness of solutions for nonlinear fractional dierential equations with integral boundary conditions," Fractional Differential Calculus, vol. 1, no. 1, pp. 29-43, 2011.

[12] Z. Liu and J. Sun, "Nonlinear boundary value problems of fractional differential systems," Computers $\mathcal{E}$ Mathematics with Applications, vol. 64, no. 4, pp. 463-475, 2012.

[13] C. F. Li, X. N. Luo, and Y. Zhou, "Existence of positive solutions of the boundary value problem for nonlinear fractional differential equations," Computers \& Mathematics with Applications, vol. 59, no. 3, pp. 1363-1375, 2010.

[14] X. Su, "Boundary value problem for a coupled system of nonlinear fractional differential equations," Applied Mathematics Letters, vol. 22, no. 1, pp. 64-69, 2009.

[15] F. Wang, "Anti-periodic fractional boundary value problems for non-linear dierential equations of fractional order," Advances in Difference Equations, vol. 2012, article 116, 2012.

[16] G. Wang, B. Ahmad, and L. Zhang, "Impulsive anti-periodic boundary value problem for nonlinear differential equations of fractional order," Nonlinear Analysis. Theory, Methods \& Applications, vol. 74, no. 3, pp. 792-804, 2011.

[17] J. Wang, L. Lv, and Y. Zhou, "Boundary value problems for fractional differential equations involving Caputo derivative in Banach spaces," Journal of Applied Mathematics and Computing, vol. 38, no. 1-2, pp. 209-224, 2012. 
[18] Y. Zhou and F. Jiao, "Nonlocal Cauchy problem for fractional evolution equations," Nonlinear Analysis. Real World Applications, vol. 11, no. 5, pp. 4465-4475, 2010.

[19] L. Zhang, G. Wang, and G. Song, “Existence of solutions for nonlinear impulsive fractional dierential equations of order $\alpha \in(2,3]$ with nonlocal boundary conditions," Abstract and Applied Analysis, vol. 2012, Article ID 717235, 26 pages, 2012.

[20] S. Zhang, "Positive solutions for boundary-value problems of nonlinear fractional differential equations," Electronic Journal of Differential Equations, vol. 36, pp. 1-12, 2006.

[21] B. Ahmad, J. J. Nieto, and J. Pimentel, "Some boundary value problems of fractional differential equations and inclusions," Computers \& Mathematics with Applications, vol. 62, no. 3, pp. 1238-1250, 2011.

[22] B. Ahmad and S. K. Ntouyas, "Fractional differential inclusions with fractional separated boundary conditions," Fractional Calculus and Applied Analysis, vol. 15, no. 3, pp. 362-382, 2012.

[23] M. Benchohra, S. Djebali, and S. Hamani, "Boundary-value problems of differential inclusions with Riemann-Liouville fractional derivative," Nonlinear Oscillations, vol. 14, no. 1, pp. 6-20, 2011.

[24] A. Cernea, "On a multi point boundary value problem for a fractional order dierential inclusion," Arab Journal of Mathematical Sciences. In press.

[25] A. Cernea, "A note on the existence of solutions for some boundary value problems of fractional differential inclusions," Fractional Calculus and Applied Analysis, vol. 15, no. 2, pp. 183-194, 2012.

[26] Y.-K. Chang and J. J. Nieto, "Some new existence results for fractional differential inclusions with boundary conditions," Mathematical and Computer Modelling, vol. 49, no. 3-4, pp. 605-609, 2009.

[27] J. Wang and Y. Zhou, "Existence and controllability results for fractional semilinear differential inclusions," Nonlinear Analysis. Real World Applications, vol. 12, no. 6, pp. 3642-3653, 2011.

[28] S. Hu and N. S. Papageorgiou, Handbook of Multivalued Analysis, Volume I: Theory, vol. 419 of Mathematics and its Applications, Kluwer Academic Publishers, Dordrecht, The Netherlands, 1997.

[29] A. Lasota and Z. Opial, "An application of the Kakutani-Ky Fan theorem in the theory of ordinary differential equations," Bulletin de l'Académie Polonaise des Sciences, vol. 13, pp. 781-786, 1965.

[30] A. A. Kilbas, H. M. Srivastava, and J. J. Trujillo, Theory and Applications of Fractional Differential Equations, vol. 204 of North-Holland Mathematics Studies, Elsevier Science, Amsterdam, The Netherlands, 2006.

[31] A. Granas and J. Dugundji, Fixed Point Theory, Springer Monographs in Mathematics, Springer, New York, NY, USA, 2003.

[32] H. Covitz and S. B. Nadler Jr., "Multi-valued contraction mappings in generalized metric spaces," Israel Journal of Mathematics, vol. 8, pp. 5-11, 1970.

[33] A. A. Tolstonogov, "A theorem of Bogolyubov with constraints generated by a second-order evolutionary control system," Izvestiya: Mathematics, vol. 67, no. 5, 2003.

[34] A. Bressan and G. Colombo, "Extensions and selections of maps with decomposable values," Studia Mathematica, vol. 90, no. 1, pp. 69-86, 1988.

[35] C. Castaing and M. Valadier, Convex Analysis and Measurable Multifunctions, vol. 580 of Lecture Notes in Mathematics, Springer, Berlin, Germany, 1977. 


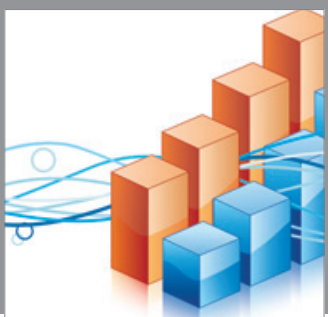

Advances in

Operations Research

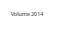

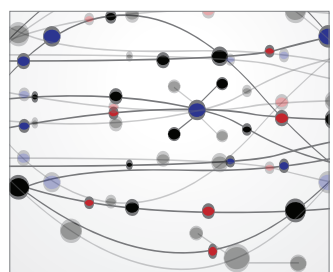

\section{The Scientific} World Journal
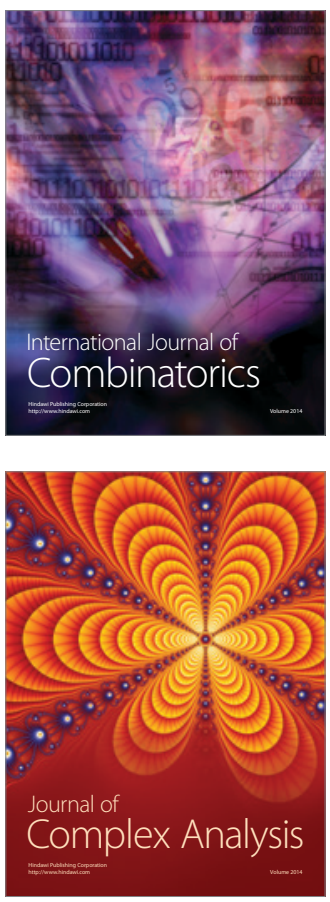

International Journal of

Mathematics and

Mathematical

Sciences
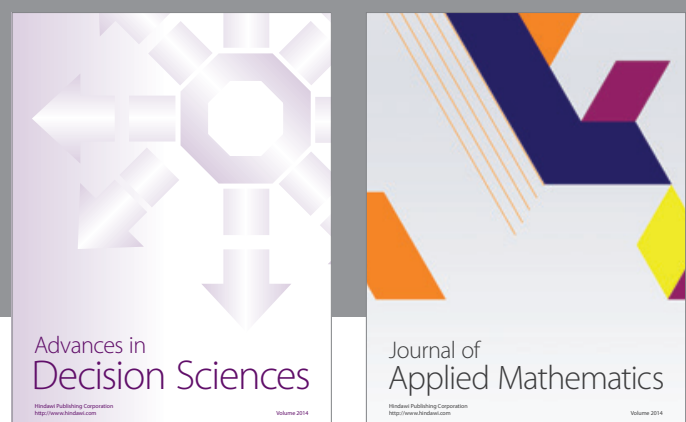

Journal of

Applied Mathematics
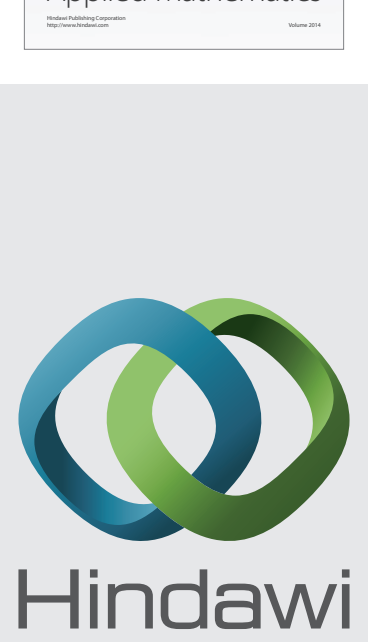

Submit your manuscripts at http://www.hindawi.com
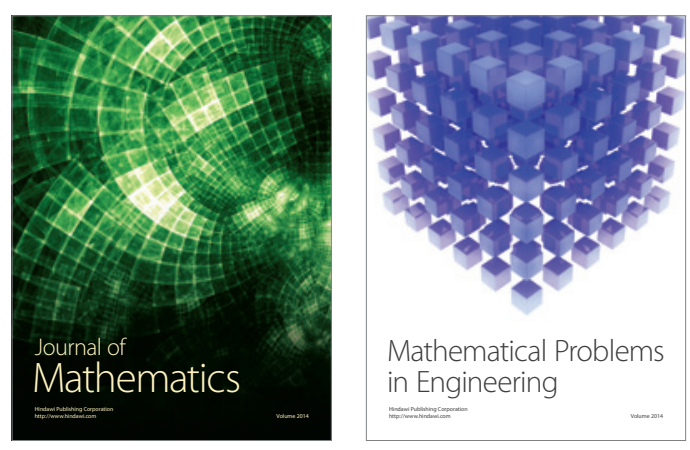

Mathematical Problems in Engineering
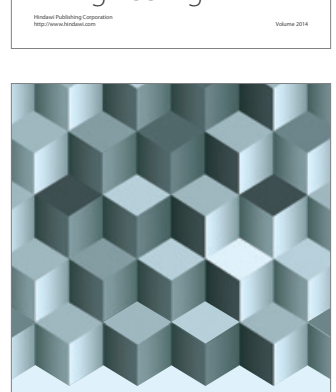

Journal of

Function Spaces
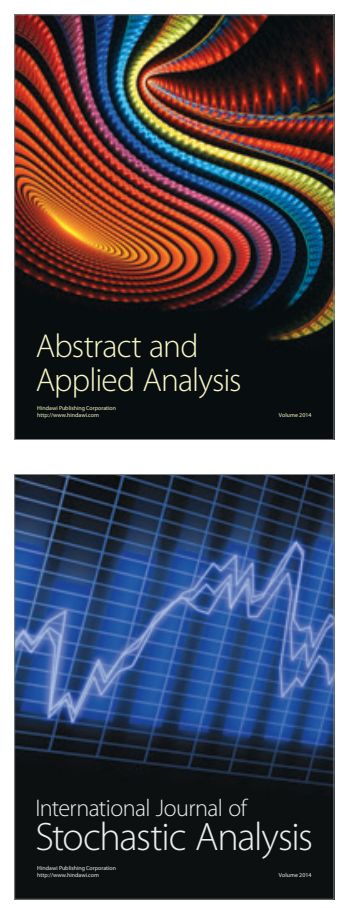

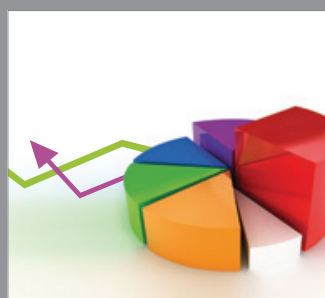

ournal of

Probability and Statistics

Promensencen
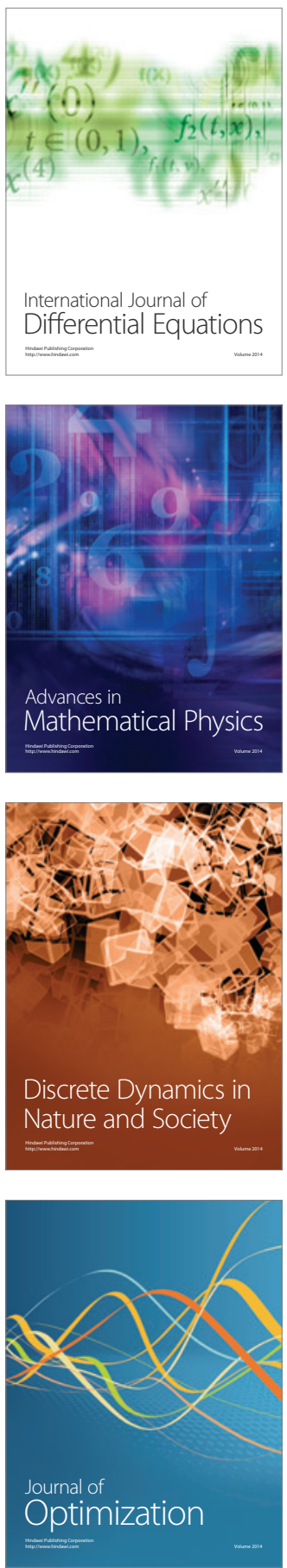